\title{
La Mejora de Procesos de Software a través de la Gestión de Procesos, el Outsourcing y la Adopción de Nuevas Tecnologías de Información y Comunicación
}

\section{Software Process Improvement in Organizations through Process Management, Outsourcing and Adoption of Information and Communication Technologies}

\author{
Jezreel Mejia ${ }^{1}$, Mirna Muñoz ${ }^{1}$, Álvaro Rocha ${ }^{2}$ \\ \{jmejia, mirna.munoz\}@cimat.mx, amrrocha@gmail.com \\ ${ }^{1}$ Centro de Investigación en Matemáticas- Unidad zacatecas, Av. Universidad No. 222, 98068, Zacatecas, \\ México \\ 2 Universidade de Coimbra, Departamento de Engenharia Informática, Pólo II - Pinhal de Marrocos, \\ 3030-290 Coimbra, Portugal
}

DOI: 10.4304/risti.e1.vii-xi

\section{Introducción}

Nos es muy grato participar en la edición de este número especial de la Revista Ibérica de Sistemas y Tecnologías de la Información (RISTI), dedicado a la temática "La Mejora de Procesos Software a través de la Gestión de Procesos, el Outsourcing y la Adopción de Nuevas Tecnologías de Información y Comunicación”. Los artículos presentados en este número especial fueron seleccionados de los artículos aceptados en el segundo Congreso Internacional de Mejora de Procesos Software CIMPS 2013, realizado en la Ciudad de Zacatecas, México del 2 al 4 de Octubre del 2013.

La Mejora de Procesos Software a través de la Gestión de Procesos, el Outsourcing y la Adopción de Nuevas Tecnologías de Información y Comunicación

La mejora de procesos software es un mecanismo importante para impulsar la competitividad y eficiencia en las organizaciones de software (Muñoz M., Mejia M, Calvo-Mazano J.A. \& San Feliu T., 2013; Mishra, 2009; Garcia I. and Pacheco C., 2009). Actualmente las organizaciones de desarrollo de software han apostado por la implementación de modelos y estándares con el objetivo de aumentar y demostrar su capacidad para incrementar la calidad de sus productos (Muñoz M, Mejia J, 2013). Por 
otra parte, estas organizaciones también se ha interesado por la aplicación de modelos para la evaluación y mejora de los procesos de desarrollo de software (Mas, A., Amengual, E. \& Mesquida, A. L., 2010; Muñoz M, Mejia J, 2013), como pueden ser CMMI (SEI, 2010) o el estándar internacional ISO/IEC 15504 (ISO, 2003; ISO, 2004; ISO, 2006; ISO, 2008), por mencionar algunos.

Este tipo de organizaciones, además de seguir reconociendo la importancia de mejorar sus procesos de desarrollo de software, han puesto especial atención hacia nuevos dominios de interés, como pueden ser el desarrollo de software en entornos Outsourcing y la adopción de nuevas tecnologías de Información y Comunicación.

En el contexto del desarrollo de software en entornos Outsourcing, aunque el mercado global de Outsourcing de Tecnologías de la Información (TI) creció en 2013 a un ritmo del 2,8\%, ligeramente superior al de 2012, se pronostica un crecimiento constante del 4,5\% hasta el 2015 (Gartner, 2013; Mejia J, García A., Muñoz M., 2013). En este tipo de proyectos se genera ciertos problemas que de acuerdo a un estudio realizado por (Deloitte, 2011) son problemas que las organizaciones cliente en su relación con los proveedores se genera como: deficiente entrega de informes, falta de transparencia y pobre comunicación. Aunque en los últimos años, han aparecido algunos modelos y estándares que contienen buenas prácticas, éstos no gestionan de una manera correcta, la relación entre el cliente y el proveedor. Ya que estos modelos y estándares solo indican el "Qué", sin indicar el "Cómo", esto último es fundamental para el desarrollo adecuado de proyecto de outsourcing y por consiguiente que las expectativas de ambos sean alcanzadas.

Con respecto a la adopción de tecnologías de información y comunicación (TICs) cualquier organización sin importar el tamaño y sector (desarrollo, comercial, educativo, industrial, etc.) la adopción de TICs permite a las organizaciones mejorar el acceso a información valiosa, aumentar el conocimiento interno y externo a ellas, mejorar las relaciones con clientes y proveedores, colaborar con otras organizaciones. Además, aumentan su eficiencia, ofrecen nuevos canales de comunicación y distribución y reducen costos de producción (Qureshi, Kamal, \& Wolcott, 2009), (Parida, Johansson, Ylinenpää, \& Baunerhjelm, 2010). Asimismo, permite la automatización y divulgación de su propio conocimiento para dar formación a su staff o clientes internos o externos. Sin embargo, aunque la adopción, creación y el uso de las TICs, están ampliamente extendidas en el mundo y han demostrado su utilidad para apoyar las estrategias de competitividad de cualquier organización (Parida et al., 2010), (Cudanov, Jasko, \& Savoiu, 2010), las empresas denominadas Pymes de cualquier sector, tienden a no adoptar las TICs para impactar en su entorno y ofrecer las ventajas mencionadas anteriormente. Por lo tanto, es fundamental encontrar formas, modelos, metodologías, nuevos paradigmas que permitan la adopción de las TICs en las Pymes.

Los artículos publicados en este número especial de RISTI tratan de cubrir la implementación de mejora de procesos, el establecimiento de una buena relación entre cliente/proveedor para la gestión en proyectos Outsourcing y como puede llevarse a cabo la adopción de TICs en organizaciones de diferente sector. 


\section{Estructura}

Los 8 artículos presentados en este número especial fueron seleccionados del total de artículos aceptados en el congreso CIMPS 2013. Este congreso ha tenido una taza de aceptación del $30 \%$ de los artículos enviados.

En el primer artículo se presenta una comparativa entre teoría y realidad de la caracterización de necesidades que presentan las Pymes para implementar una mejora de procesos de software exitosa. Este estudio se presenta por la necesidad actual en el ámbito del desarrollo de software a nivel mundial hacia las Pymes que ocupan un considerable porcentaje del número de empresas generando el $52 \%$ del producto interno bruto de los países y alrededor del $72 \%$ de los empleados formales.

El segundo artículo propone un método que guie a las Pymes en la Mejora de Procesos Software para definir y establecer los objetivos de negocio y la identificación de las mejores prácticas internas de la organización para entender su forma de trabajo a través de la extracción del conocimiento tácito. Debido que en la actualidad es muy común que las Pymes no efectúen la recolección de datos y tampoco tengan procesos definidos.

En el tercer artículo se aborda un estudio de elementos y aspectos comunes entre estándares ISO, presentando un marco que facilita la implantación integrada de éstos estándares enfocados en la gestión de Tecnologías de Información (TI). Con la finalidad de que las empresas de desarrollo de software además de la necesidad de mejorar sus procesos de desarrollo de software, también puedan aumentar la capacidad de los procesos de otras disciplinas, como pueden ser la gestión de servicios de TI o la gestión de la seguridad de la información.

En el cuarto artículo se enfoca en el contexto del Outsourcing por la falta de una buena relación que debe establecerse entre un cliente y un proveedor de servicios para lograr las expectativas de los acuerdos. Por lo tanto, este artículo presenta una herramienta nombrada Quetzalcoatl, con el objetivo de generar y evaluar contratos para proyectos de desarrollo de software en entornos Outsourcing como apoyo a las Pymes, para lograr establecer una buena relación mediante la formalización de las expectativas del cliente a través de un contrato como alternativa.

Siguiendo el contexto del Outsourcing el quinto articulo presenta una metodología que guíe a los proveedores a lo largo de todo el ciclo de vida del Outsourcing y facilite la provisión de servicios de calidad bien gestionados. Aun cuando el mercado de Outsourcing ha estado creciendo en los últimos años existen muchos fracasos de proyectos. Estos fracasos se han debido en gran parte a los problemas con los proveedores, aspecto en el que se enfoca la metodología que aborda este artículo.

Abordando la adopción de TICs, en el Sexto artículo se presenta en el contexto de las redes de comunicación no intrusivas una propuesta tecnológica de comunicación. Dicha propuesta se desarrolla para la comunicación entre dos personas. Se incluye también una propuesta para escalar dicha comunicación para su uso en pequeñas redes sociales (familias) sin que por ello crezcan desproporcionadamente las posibilidades de intrusión. 
En el Séptimo artículo se presenta el uso del Modelo del Usuario Perezoso, para explicar la adopción de Tecnologías de Información en Pequeñas Empresas. Ya que una forma de dar soporte al desarrollo y crecimiento de organizaciones de todo tamaño, es mediante el uso de Tecnologías de Información. Debido al importante papel de las Pequeñas Empresas en las economías de todo el mundo, la investigación sobre la adopción de estas tecnologías se vuelve fundamental.

Y finalmente en el octavo artículo se identifican los factores determinantes para el éxito de la adopción de nuevas tecnologías de comunicación en el contexto de cursos online abiertos y masivos que surge de la combinación de e-Learning y modelos de redes sociales, y que se ha integrado en muchos programas educativos transformando las organizaciones e-Learning a nivel mundial. Para lograr este estudio se realiza la revisión de la literatura científica, y estadísticas propias 35 plataformas con más de 7.000 cursos.

\section{Agradecimientos}

Agradecemos a la AISTI (Asociación Ibérica de Sistemas e Tecnologías de Información) por darnos la oportunidad de dar a conocer los trabajos más relevantes presentados en el congreso CIMPS 2013. Asimismo, expresamos nuestra gratitud a los autores de los trabajos seleccionados, lo que hizo posible este número especial en RISTI.

\section{Referencias}

Muñoz M., Mejia M, Calvo-Mazano J.A. \& San Feliu T. (2013). Involvement of Stakeholders in Software Processes Improvement to Reduce Change Resistance, EuroSpi, CCIS 364, pp, 202-2013, Springer-Verlag Berlin Heidelberg

Mishra, D., Mishra, A. (2009). Software Process Improvement in SMEs: A Comparative View. Computer Science and Information Systems, Vol. 6, No. 1, 111-140, (2009).

Garcia I. and Pacheco C., Toward Automated Support for Software Process Improvement Initiatives in Small and Medium Size Enterprises", R. Lee \& N. Ishii (Eds.): Soft. Eng. Research. Manage. \& Appli. 2009. SCI 253. pp. 51-58. (2009)

Mirna M, Jezreel M, Establishing Multi-model Environments to Improve Organizational Software Processes, Advances in Intelligent Systems and Computing Volume 206, pp 445-454 (2013).

Mas, A., Amengual, E. \& Mesquida, A. L. (2010). Application of ISO/IEC 15504 in Very Small Enterprises. Systems, Software and Services Process Improvement, Communications in Computer and Information Science 99, Springer-Verlag, 290301.

Mejia J, García A,, Muñoz M. (2013). TSPi to Manage Software Projects in Outsourcing Environments, Advances in Intelligent Systems and Computing Volume 206, pp 411-420.

Gartner. (2013). Global IT outsourcing market to touch $\$ 288$ billion in 2013. The Times of India. 
Deloitte. (2011). The Outsourcing Vendor Management Program Office (VMPO): Art, science, and the power of perseverance. New York: Deloitte Consulting LLP.

SEI (2010). CMMI $®$ for Development, Version 1.3, Software Engineering Institute.

ISO (2003). ISO/IEC 15504-2:2003 Information technology -- Process assessment -Part 2: Performing an assessment.

ISO (2004). ISO/IEC 15504-1:2004 Information technology -- Process assessment -Part 1: Concepts and vocabulary.

ISO (2006). ISO/IEC 15504-5:2006 Information Technology - Process Assessment Part 5: An exemplar Process Assessment Model, International Organization for Standarization.

ISO (2008). ISO 9001:2008 Quality management systems - Requirements, International Organization for Standarization.

Qureshi, S., Kamal, M., \& Wolcott, P. (2009). Information Technology Interventions for Growth and Competitiveness in Micro-Enterprises. International Journal of EBusiness Research (IJEBR), 5(1), 117-140.

Parida, V. ., Johansson, J., Ylinenpää, H. ., \& Baunerhjelm, P. (2010). Barriers to information and communication technology adoption in small firms. Retrieved from http://entreprenorskapsforum.se/swe/wpcontent/uploads/2010/09/WP_Barriers_to_ICT_adoption.pdf

Cudanov, M., Jasko, O., \& Savoiu, G. (2010). Interrelationships of Organization Size and Information and Communication Technology Adoption. Journal of Applied Quantitative Methods, 5(1). 